\title{
LA LETTERATURA ITALIANA TRADOTTA IN BRASILE: INDAGINE E CONSIDERAZIONI SUL PANORAMA CONTEMPORANEO E ULTRACONTEMPORANEO
}

\author{
Adriana Iozzi Kuein \\ LUCIA W WTAGHIN
}

\begin{abstract}
Lo scenario della letteratura italiana contemporanea tradotta in Brasile comprende autori e titoli consacrati dall'editoria perché ormai "classici" o in quanto campioni di vendite, ma anche giovani autori e nuove opere, spesso pubblicati da editori minori. L'articolo è dedicato a tracciare a grandi linee il panorama attuale, con particolare attenzione per le opere italiane arrivate più recentemente sul mercato brasiliano.
\end{abstract}

PAROLE CHIAVE: letteratura italiana tradotta; Brasile; Italia; Novecento

RESUMO: O panorama da literatura italiana traduzida no Brasil abrange autores e títulos consagrados pelos editores porque já "clássicos" ou porque campeões de venda, mas também jovens autores e novas obras, muitas vezes publicados por editores menores. $O$ artigo é dedicado a traçar em grandes linhas o panorama atual, com especial atenção pelas obras italianas que chegaram mais recentemente ao mercado brasileiro.

PALAVRAS-CHAVE: literatura italiana traduzida; Brasil; Itália; Novecentos

* Universidade de São Paulo, São Paulo (Brasil) - adriozzi@terra.com.br / luciawataghin@gmail.com Processo n.2013/20971-0 Fundação de Amparo à Pesquisa do Estado de São Paulo (FAPESP)

DOI: http://dx.doi.org/10.11606/issn.2238-8281.v0i34p43-53 
ABSTRACT: The scenario of contemporary Italian literature translated in Brazil includes not only authors and titles acclaimed by publishers as they are now "classics" or bestsellers, but also young authors and new works, usually published by smaller publishing houses. This article seeks to provide an outline of the current panorama, placing special emphasis on the Italian works which have recently reached the Brazilian market.

KEYWORDS: translated Italian literature - Brazil - Italy - 20th Century 


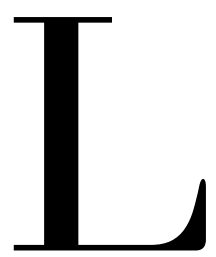

o spazio d'identità della letteratura italiana in Brasile, non diversamente da quello che succede in altri paesi, si definisce entro confini costruiti intorno alle politiche editoriali di livello globale, in cui gli agenti letterari, i mediatori culturali e i traduttori hanno un ruolo rilevante ${ }^{1}$.

Il mercato editoriale brasiliano, è importante ricordarlo, si è rinnovato completamente a partire dalla fine degli anni '80, dopo la caduta del regime militare, fatto che ha generato, tra l'altro, una grande apertura riguardo alle scelte editoriali nel paese. Da allora si è allargato significativamente il numero degli autori italiani tradotti, soprattutto contemporanei, e la lunga e molteplice lista ${ }^{2}$ comprende nomi e titoli consacrati dall'editoria perché ormai "classici" o in quanto campioni di vendite, ma anche giovani autori e nuove opere, spesso pubblicati da editori minori.

1 Questo articolo è stato pubblicato, con piccole variazioni, nella rivista "narrativa", nuova serie, n. 38 - 2016, Paris, Presses universitaires de Paris Nanterre

2 In questo elenco troviamo autori di ogni tipo: da Calvino a Eco, da Tabucchi a Tamaro, Baricco, Manganelli, Primo Levi, Castiglione, Del Giudice, Fenoglio, Svevo, Pirandello, Leopardi, Montale, Moravia, Natalia Ginzburg, Buzzati, Pavese, Pasolini, Magris, Vittorini, Busi, Brancati, Bufalino, e altri ancora, per arrivare ai recentissimi Camilleri, Ammaniti, Veronesi, Ferrante. Tra i saggisti spiccano Croce, De Sanctis, Gramsci, Norberto Bobbio, Argan, Nicola Abbagnano, Giorgio Agamben, Roberto Calasso, Pietro Citati, Carlo Ginzburg e più recentemente Mario Perniola, Franco Moretti, Alfonso Berardinelli e Remo Ceserani, per citarne alcuni. 
Complesso e problematico, il fenomeno delle traduzioni di opere italiane non è stato ancora esaustivamente analizzato dagli studiosi brasiliani, ma sarebbe opportuno illustrare la funzione tutt'altro marginale che questa attività svolge nella disseminazione, ricezione e trasformazione di tematiche, generi, stili e poetiche, facendo riferimento a iniziative che hanno messo a fuoco aspetti legati alla diffusione del lavoro di autori italiani nel quadro della produzione letteraria globale più recente, senza dimenticare il ruolo delle istituzioni accademiche brasiliane che continuano (ancora) a formare lettori e studiosi di italianistica. Su questo fronte, merita attenzione il lavoro che stiamo svolgendo, frutto di una cooperazione tra due gruppi di ricercatori legati alle università brasiliane di São Paulo e di Santa Catarina, impegnati sin dal 2010 in un progetto di mappatura delle opere di letteratura italiana tradotte in Brasile.

Uno dei primi risultati di questo progetto è stata la creazione di un catalogo elettronico di libri italiani tradotti intitolato Dicionário da Literatura Italiana Traduzida. La bibliografia riportata nel Dizionario elenca gli autori e le opere italiane pubblicati in Brasile dal 1900 fino ad oggi, divisi in due parti: una prima che comprende la letteratura italiana tradotta dal 1900 al 1950, disponibile sul sito dell'Universidade Federal de Santa Catarina (www.dlit.ufsc.br), e una seconda, ancora in fase di elaborazione, che riporta la letteratura italiana tradotta dal 1950 in poi, i cui risultati si trovano già disponibili sul sito dell'Universidade de São Paulo (http://www.usp.br/dlit).

Questa proposta di mappatura della letteratura italiana tradotta, con l'ausilio di un dizionario elettronico delle opere, costituisce un esercizio di quel distant reading, definito da Franco Moretti come necessario per cercare di costruire una storia letteraria "più razionale" (Moretti, 2005, p. 10), giacché è impossibile un close reading individuale di intere letterature. Partiamo dalla constatazione, che ritroviamo in Moretti ma che è già in partenza connaturata alla nostra ricerca, che siamo di fronte a un campo vastissimo, che può essere letto e radiografato solo a patto di poter contare su vaste cooperazioni, su strumenti e dati raccolti da molti e con l'aiuto di altre scienze e discipline (vedi anche Lambert, in Guerini et alii, 2011, p. 11).

La storia letteraria che stiamo cercando di ricostruire è quella dell'incontro della letteratura italiana tradotta con il suo contesto d'arrivo in Brasile, cioè con i lettori, gli editori, i critici e gli autori brasiliani. Osservare quali e quanti autori e titoli sono stati tradotti, e come, e in che modo sono stati presentati al nuovo pubblico, può aprire diversi campi di riflessione: sui rapporti di forza fra lingue e letterature, sui processi di egemonia (Venuti, Berman, Lambert), ossia sui rapporti fra potere/istituzioni/ideologia e letterature, o sul legame tra letteratura e 
identità nazionale, o sul canone e sulla capacità di autoconservazione dell'alta letteratura, o sulla quantità di "letteratura di consumo" che è stata immessa su un dato mercato, o ancora, sulla stessa distinzione fra "alta letteratura" e "letteratura di consumo", che pare sull'orlo dell'estinzione. Ci si domanda inoltre se la penetrazione di certi autori o certi temi o tendenze nel mercato dipenda da una sintonia preesistente tra i due sistemi culturali, o se si tratti piuttosto, ma forse in casi diversi, di fenomeni globali a cui nessuna cultura resiste. Se Eco, Calvino e Saviano, per esempio, vendono molto non solo in Brasile, ma dappertutto, ne dedurremo l'esistenza di un terreno comune globale, e non specifico, su cui attecchiscono determinati prodotti culturali; mentre fenomeni di vendita meno clamorosi indicano la presenza di segmenti di lettori con interessi specifici e più circoscritti. In ogni caso entrambi i fenomeni interessano anche per quanto possono dirci sul contesto ideologico del sistema ricevente, che accoglie certi autori e certe idee e non altri.

Il nostro è uno studio di quantificazione, e il distant reading ci permette di osservare " $\mathrm{i}$ rapporti, i pattern, le forme" (Moretti, 2005, p. 3). Dai nostri dati sorgono ipotesi di nuovi rapporti, pattern, forme. Le indagini possono partire dai dati sulla presenza di certi autori e titoli in Brasile (verifichiamo per esempio che Tabucchi e Calvino sono autori molto tradotti e da questo fatto partiamo per indagare sui loro motivi d'affinità con la cultura ricevente e osservare il loro impatto sull'ospitale contesto d'arrivo), oppure, viceversa, partiamo da pattern già individuati nella cultura di partenza per verificare che ne è stato all'arrivo nel nuovo sistema.

Partendo da pattern già individuati, sia in saggi e articoli critici che in manuali vecchi e nuovi di storia della letteratura, abbiamo provato per esempio a dividere scrittori e opere per gruppi o per periodi per cercare di capire in che misura si scompongono o si mantengono nella letteratura d'arrivo le reti di rapporti iniziate nella letteratura d'origine. Abbiamo anche cercato di verificare cosa succede ai singoli autori, a gruppi o tendenze, in momenti storici coincidenti (per esempio, si sa che al dannunzianesimo e al clima decadentista/wildiano di fine ottocento e inizio novecento corrisponde un clima analogo in Brasile, specialmente nel sud, con lo scrittore João do Rio, o che ci sono stretti e importanti rapporti tra l'Arcadia italiana e un movimento poetico ad essa ispirato, e che porta lo stesso nome, nel Minas Gerais, nel Settecento) o più distanti tra loro nel tempo, ma radicalmente connessi (come i movimenti delle avanguardie europee di inizio novecento e il modernismo brasiliano degli anni venti).

Sono stati fatti studi su casi particolari che illustrano le fluttuazioni prevedibili ma pur sempre notevoli dei canoni (è il caso di Papini, di cui sono stati tradotti ben dieci titoli tra il 1951 e il 
1963, ma che poi è scomparso, con l'eccezione di un'unica traduzione nel 1993, di I testimoni della passione, dell'Ediouro di Rio de Janeiro), o fenomeni di manipolazione editoriale, come lo strano caso dell' ambizioso romanzo Il mulino del Po, di Riccardo Bacchelli, pubblicato negli anni '50 in Brasile, ma spezzato in due volumi da vendere separatamente.

Un altro caso interessante è quello di un titolo come Cuore, fino agli anni settanta uno dei più forti best seller della letteratura per l'infanzia in Brasile (dove il suo autore fu conosciuto anche per i libri di viaggi, usciti in edizioni illustrate, di lusso, all'inizio del novecento), ma non più ripubblicato fino al 2011, quando finalmente esce in nuova traduzione, corredata di postfazione e bibliografia rivolte non all'infanzia, ma al lettore adulto e sofisticato. E ci sono naturalmente invece autori come Pirandello (conosciuto in Brasile molto più di Svevo), la cui opera teatrale e narrativa è costantemente presente e periodicamente ritradotta. Una curiosità: il best seller degli anni '70 Porci con le ali (1976), di Marco Lombardo Radice e Lidia Ravera, tradotto e pubblicato in Brasile dalla Brasiliense, è arrivato alla nona edizione del 1986 e se ne trovano ancora oggi ${ }^{3}$ più di 200 esemplari nel mercato online dell'usato.

Le reti di rapporti che si stabiliscono sono naturalmente fluttuanti, dipendenti da molteplici fattori e, in ultima analisi, da letture soggettive. Ad esempio l'antologia Neorrealismo italiano. Raizes populistas, curata da una docente universitaria (Terenzi Vicentini, 2010, uscita postuma e derivata da una tesi di master discussa all’Università di São Paulo nel 1979), riunisce quattro autori italiani tradotti in portoghese: Pavese, Vittorini, Pratolini, Carlo Levi. E negli anni immediatamente successivi alla discussione della tesi escono ben quattro traduzioni brasiliane di Pavese, ${ }^{4}$ oltre a Cronache di poveri amanti (1983) di Pratolini e Cristo si è fermato a Eboli (1986) di Carlo Levi, ma curiosamente neanche un titolo di Vittorini.

Forse non a caso la letteratura neorealista italiana, antifascista e di sinistra, vive una fase fortunata in Brasile proprio negli anni della fine della dittatura; d'altra parte tale fortuna è sicuramente dipendente dalla concomitante fortuna italiana, e legata agli studi di italianistica nelle università brasiliane, che a loro volta sono strettamente connessi alla critica italiana. Difatti tutti i titoli pubblicati negli anni '80 sono tradotti da docenti dell'Università di São Paulo. Una nuova ondata di simpatia per gli stessi autori si ritrova negli anni 2000 - e ciò fa pensare che effettivamente sia utile considerarli in gruppo -, grazie alla fervida ed energica

3 Dati di maggio 2016.

4 La luna e i falò (1986), La bella estate (1987), Tra donne sole (1988), Il mestiere di vivere (1988). 
attività editoriale della Cosac Naify. Quanto a Fenoglio, quello che è considerato il suo miglior libro, Una questione privata, sarà pubblicato per la prima volta solo nel 2001, dalla Berlendis.

Anche la fortuna brasiliana di Giorgio Bassani inizia solo negli anni ' 80 (benché il film di De Sica, che diede notorietà mondiale anche al libro, fosse già uscito nel 1970) con $I l$ giardino dei Finzi-Contini (1980 e 1987), e riprende negli anni duemila, con un solo titolo, Gli occhiali d'oro (2002). Si nota però che molti titoli di narrativa italiana godono della fama riflessa dei film da loro tratti: il film di Visconti Il gattopardo (1963) è molto probabilmente la ragione scatenante dell'interesse internazionale anzitutto per il romanzo omonimo $(1974,1987$, 2003), ma anche per altri titoli di Tomasi di Lampedusa (raccolte di racconti uscite negli anni 1980, 1997, 2002). Un altro autore conosciuto in Brasile grazie al cinema è Vitaliano Brancati: il romanzo Il bell'Antonio, del 1949, sarà tradotto solo nel 1962, dopo l'uscita del film di Bolognini, nel 1960. Seguono le traduzioni degli altri romanzi: Don Giovanni in Sicilia (1989) e Paolo il caldo (1991). Da ricordare ancora Camillo Boito, il cui Senso esce in traduzione nel 1990 (il film di Visconti è del 1954). Sempre di più l'esistenza di trasposizioni filmiche costituisce un forte stimolo alle traduzioni: due titoli di Niccolò Ammaniti (Che la festa cominci e Io e te) sono stati tradotti e pubblicati dalla Bertrand Brasil (rispettivamente nel 2012 e 2013), dopo che cinque dei suoi romanzi erano stati trasposti in film. ${ }^{5}$

\section{Autori del Novecento ancora in circolazione nel mercato librario brasiliano}

Tra gli autori del Novecento ancora in circolazione nelle librerie negli anni duemila è da ricordare Natalia Ginzburg, che gode di una sorta di revival editoriale grazie alle edizioni curatissime della Cosac Naify (Lessico famigliare, 2005, Caro Michele, 2009, Le piccole virtù, 2015), con apparato critico eccellente, in alcuni casi scritto ad hoc (da Ettore Finazzi-Agrò per Lessico famigliare, e da Vilma Arêas per Caro Michele), della Berlendis (̇̀ stato così, 2001) e della José Olympio di Rio de Janeiro (Famiglia, 2003). Responsabili del lancio del fino a allora inedito Vittorini sono ancora la Cosac Naify, con Conversazione in Sicilia (2005), Uomini e no (2007), Sardegna come un'infanzia (2011), e la Berlendis, con Erica e i suoi fratelli (2001).

Altre presenze molto forti sono Primo Levi, con sette titoli tradotti e pubblicati da editori importanti, tre dei quali negli anni duemila, Pasolini con sei titoli tradotti, Calvino con più di quindici, Umberto Eco con nove titoli, Buzzati con almeno sette titoli, oltre al Poema a fumetti,

5 L'ultimo capodanno, Branchie, Io non ho paura, Come Dio comanda e Io e te. 
tradotto nel 2010. La presenza di Calvino, verificata anche nella stampa di grande circolazione, nei corsi universitari, nelle numerose tesi a lui dedicate, soprattutto a partire dagli anni ' 90 , è veramente immensa. Un discorso a parte va fatto per Tabucchi, che arriva in Brasile anche un po' grazie al suo interesse per la letteratura e la storia portoghese e alle sue traduzioni del poeta brasiliano Carlos Drummond de Andrade: nove titoli tradotti in Brasile, tra il 1998 e il 2013. Il decimo libro di Tabucchi ancora in circolazione nelle librerie è Requiem, scritto direttamente in portoghese. Un ultimo nome tra gli ipertradotti: Alessandro Baricco, poco amato dalla critica italiana per il suo "falso sperimentalismo" (Donnarumma 2014, p. 56), per l' "insistente" autocompiacimento, per la "superficiale musicalità che si atteggia a profondità" (Ferroni 2015, p. 307), ha sfondato qui molte barriere, con undici libri tradotti, dal 1999 a oggi.

Molti classici sono ormai fuori catalogo: quasi scomparso Moravia, per esempio, che pure fu tradottissimo dagli anni '50 ai '90 (circa venticinque titoli tradotti); nelle librerie oggi si trovano solo i racconti, editi da Berlendis nel 2003, e l'atipico Storie della preistoria, edito dall'Editora 34 nella collana Teen. Fuori catalogo anche Giovanni Arpino, Vasco Pratolini, Curzio Malaparte, Gadda.

Fin qui si è parlato di opere e autori di nicchia, proposti e curati da editori che si dedicano soprattutto o esclusivamente a titoli di "alta cultura", come la Cosac Naify o la Berlendis, che si occupa in particolare della letteratura italiana a cui ha dedicato una collana esclusiva. Alcuni classici italiani come Dante, Boccaccio e Machiavelli si pongono invece al di là delle vendite di nicchia: sono "titoli globali", continuamente ritradotti, che hanno conquistato un mercato stabile o addirittura crescente in certi periodi, grazie a nuove edizioni tascabili, vendute anche nelle edicole.

\section{Tempi di globalizzazione}

Pensiamo ora ai cambiamenti più recenti. A partire dagli anni ' 80 la situazione del mercato librario è cambiata in tutto il mondo per effetto appunto della globalizzazione dell'industria della comunicazione. Tra le conseguenze più notevoli di questo processo, Benvenuti e Ceserani indicano una polarizzazione nell'industria editoriale: da una parte la concentrazione della produzione libraria nelle mani di grandi editori, portatori di ideologie e concezioni della letteratura bene identificabili, e dall'altra la proliferazione di piccoli editori, la cui strategia editoriale è volta alla vendita immediata quale unico, o quasi, parametro di selezione dei libri da pubblicare (Benvenuti e Ceserani, 2012, p. 143). In questo quadro si osserva il rafforzamento della tendenza all'annullamento delle distinzioni fra alta e bassa letteratura. Queste ultime sono 
categorie ancora utili per lettori e editori, ma effettivamente nella marea di titoli sconosciuti che arrivano sul mercato, proposti da piccoli editori, pare molto difficile raccapezzarsi.

Per quanto riguarda il Brasile, la situazione è simile a quella del resto del mondo e i libri più venduti sono sempre quelli globali ${ }^{6}$. La nuova geografia delle centralità a livello globale comprende anche San Paolo (Fiorentino, 2007, apud Ceserani e Benvenuti, 2012, p. 146) e da qui le vendite si irradiano nelle altre città brasiliane. Una rapida ricerca nel mercato brasiliano on line dei libri usati rivela la presenza di almeno 166 titoli di Camilleri (ivi compresi alcuni titoli in italiano e in altre lingue, ma anche moltissime traduzioni in portoghese), uno dei quali, Acqua in bocca (2013), scritto a quattro mani con Carlo Lucarelli.

Sarebbe da verificare se il mercato brasiliano è avido di letteratura poliziesca o thrillers tanto quanto lo è il mercato globale in genere, ma è un fatto che anche Almost blue di Carlo Lucarelli, pubblicato dalla casa editrice Conrad, si trova nelle bancarelle virtuali oggi in 79 esemplari in traduzione brasiliana. C'è da aggiungere che la Conrad, che si è occupata soprattutto di cultura pop, di fumetti giapponesi e coreani, è responsabile anche della coraggiosa importazione - e traduzione - di tre libri del gruppo Wu Ming, (Luther Blisset - Q, 2002, 54, 2005 e New thing , 2008). Altri due giallisti contemporanei che hanno trovato spazio nel mercato brasiliano sono Marcello Fois, con tre titoli tradotti nel 2004 e 2005 per la Coleção negra della Record, e Giorgio Faletti, anche lui con tre titoli (2010, 2011, 2012), l'ultimo dei quali è Appunti di un venditore di donne. Quanto a Roberto Saviano, il best seller Gomorra (Mondadori, 2006) nel 2008 si trovava sugli scaffali delle librerie brasiliane, tradotto e pubblicato dalla Bertrand Brasil e seguito rapidamente da altri quattro titoli: Il contrario della morte (2009), La bellezza e l'inferno (2011), La macchina del fango (2012) e Zero zero zero (2014). La campagna pubblicitaria brasiliana punta sull'immensa fama già esistente (notizie come "oltre 10 milioni di copie vendute in oltre 40 paesi" sono sufficienti per indurre all'acquisto nuovi lettori) e sulla storia personale dello scrittore, che è di per sé buon motivo di interesse e di curiosità. Roberto Saviano è autore e personaggio, e nelle campagne pubblicitarie si parla del "valore morale e civile" della sua "esistenza scomoda", del fatto che "vive nascosto da cinque anni", come è scritto nella presentazione sul sito della Saraiva, una delle più grandi catene di librerie brasiliane.

Come leggere Saviano? Donnarumma propone una lettura di Saviano confrontandolo con

6 Secondo Benvenuti e Ceserani, "gli italiani più venduti in Francia, Spagna e Germania, esclusi i grandi classici del Novecento e autori affermati come Eco e Baricco, [sono] Camilleri, Saviano, Melissa P., Moccia e autrici e autori per l'infanzia" (Benvenuti e Ceserani 2012, p. 145). 
Pasolini e facendo presente che Saviano, a differenza di Pasolini, pensa alla scrittura "in nome di una partecipazione alla vita pubblica che è post-ideologica e post-partitica" (Donnarumma 2014, p. 16). Ossia, Saviano appartiene al numero degli scrittori che si occupano di letteratura, ma anche della vita pubblica, come Pasolini, Calvino, Sciascia e, prima di loro, Pavese e Vittorini, e non a quelli che "ostentano indifferenza provocatoria per l'attualità e la vita civile" (qui Donnarumma allude agli amanti della borgesiana letteratura come menzogna) (idem, p. 20).

Post-ideologico - se si intendono qui le grandi ideologie, perché di microideologie personali o di piccoli gruppi è ancora ricco il panorama contemporaneo - e post-partitico appare tutto il momento storico attuale, in Italia e in Brasile. Anche il Brasile, come e forse anche più dell'Italia vittima della violenza organizzata e di meccanismi di potere perversi, rilegge Saviano come "un emblema della lotta contro le organizzazioni criminali" (Folha de São Paulo, 24/10/2012), in un clima di crollo definitivo della credibilità di partiti e ideologie.

Molto venduti anche in Brasile altri autori di best sellers italiani, ${ }^{7}$ alcuni dei quali mai nominati dalla critica né da manuali di letteratura, come Federico Moccia, con ben quattro titoli (Scusa se ti chiamo amore; Scusa ma ti voglio sposare; Ho voglia di te e Amore 14, tutti usciti tra il 2009 e il 2012) e 250 copie in circolazione nel mercato online dell'usato, e Melissa P. (Melissa Panarello, oggi trentenne, concorrente di reality show, un particolare che la dice lunga sulla distanza che la separa, nella concezione della vita e della letteratura, da Calvino o Pasolini o da qualunque altro autore del passato lontano e vicino, ma forse non da tutti i suoi coetanei), con due libri che sono stati grandi successi di vendita: Cento colpi di spazzola prima di andare a dormire e Tre, tradotti rispettivamente nel 2004 e nel 2011.

Un'altra forte produttrice di romanzi sentimentali già da tempo sul mercato è Susanna Tamaro, autrice del best seller Va'dove ti porta il cuore (1995), che può essere avvicinata al brasiliano Paulo Coelho per la produzione di tipo sapienziale, con tredici titoli tradotti e un numero impressionante di copie presenti nel mercato online. La presenza sul mercato online attesta l'effettiva acquisizione di questi libri, ma indica anche, curiosamente, che il loro ciclo di vita è relativamente breve. In questo caso specifico, osserviamo che nel giro di vent' anni (dal 1995, l'anno della prima edizione brasiliana di Va'dove ti porta il cuore, a oggi) i lettori di Tamaro hanno comprato e già rivenduto almeno 600 dei suoi libri usati. Molti altri

7 La breve lista degli autori più venduti presentata da Benvenuti e Ceserani non a caso è non discriminatoria: sono best sellers Camilleri e Saviano, così come i romanzi sentimentali di Melissa P. 
narratori italiani degli anni '80 e '90 sono presenti, con un numero minore di titoli tradotti, e contribuiscono a formare il quadro di una narrativa complessa, vivace, solida.

Più difficili da valutare sono un gran numero di autori tradotti, nuovi e forse ancora "minori”, di cui sappiamo poco: l'elenco è lungo (per vederlo basta dare un'occhiata al Dicionário) e non è facile districarsi nella selva dei nomi. Si tratta a volte di veri e propri best seller (o almeno, così sono definiti dalle case editrici). È il caso dei cicli avventurosi e esoterici di Valerio Evangelisti (quattro titoli negli anni 2000, di cui due dedicati al personaggio Nostradamus), o dei romanzi storici dell'antichista e giornalista scientifico Valerio Massimo Manfredi, con almeno nove titoli tradotti negli anni 1999-2008, e ancora dei romanzi storici di Andrea Frediani (in Brasile è stato tradotto Jerusalem, nel 2011).

In questa disamina del panorama della letteratura italiana tradotta non si è tenuto conto di vari generi che pure sono bene rappresentati, come il fumetto, la letteratura per bambini e ragazzi, la poesia, il saggio, che meriterebbero sicuramente dei capitoli a parte. Concludiamo così ricordando che il nostro contributo per ora non è che un primo tentativo di presentare parte dell'apparato informativo relativo a scrittori che, nel corso degli ultimi decenni, hanno conosciuto in Brasile una fortuna fondata su parametri che potranno essere sottoposti al vaglio dell'indagine critica grazie a questo tipo di lavoro.

\section{Riferimenti Bibliografici}

BENVENUTI, Giuliana; CESERANI, Remo, La letteratura nell'età globale, Bologna, Il Mulino, 2012.

DONNARUMMA, Raffaele, Ipermodernità. Dove va la narrativa contemporanea. Bologna, Il Mulino, 2014.

FERRONI, Giulio, Letteratura italiana contemporanea 1945-2014, Milano, Mondadori, 2015 (2 ed.).

GUERINI, Andréia, TORRES, Marie-Hélène Catherine, COSTA Walter Carlos, Literatura e tradução, Rio de Janeiro, 7Letras, 2011.

LA PORTA, Filippo, La nuova letteratura italiana. Travestimenti e stili di fine secolo, Torino, Bollati Boringhieri, 1995 (nuova edizione 1999).

MORETTI, Franco, La letteratura vista da lontano, Torino, Einaudi, 2005.

PETERLE, Patricia (a cura di), A literatura italiana no Brasil e a literatura brasileira na Itália: sob o olhar da tradução, Tubarão, Copiart, 2011.

PETERLE, Patricia, SANTURBANO, Andrea, WATAGHIN, Lucia (a cura di), Literatura italiana traduzida no Brasil 1900-1950, Rio de Janeiro, Comunità, 2013.

TERENZI VICENTINI, Marzia, Neorrealismo italiano. Raízes populistas, Curitiba, Segesta, 2010.

Recebido em 13/04/2017

Aprovado em 20/07/2017 